PROCEEDINGS OF THE

AMERICAN MATHEMATICAL SOCIETY

Volume 129 , Number 11, Pages 3437-3444

S 0002-9939(01)05964-0

Article electronically published on April 24, 2001

\title{
ON FILLABLE CONTACT STRUCTURES UP TO HOMOTOPY
}

\author{
PAOLO LISCA
}

(Communicated by Ronald A. Fintushel)

\begin{abstract}
Let $Y$ be a closed, oriented 3-manifold. The set $\mathcal{F}_{Y}$ of homotopy classes of positive, fillable contact structures on $Y$ is a subtle invariant of $Y$, known to always be a finite set. In this paper we study $\mathcal{F}_{Y}$ under the assumption that $Y$ carries metrics with positive scalar curvature. Using Seiberg-Witten gauge theory, we prove that two positive, fillable contact structures on $Y$ are homotopic if and only if they are homotopic on the complement of a point. This implies that the cardinality of $\mathcal{F}_{Y}$ is bounded above by the order of the torsion subgroup of $H_{1}(Y ; \mathbb{Z})$. Using explicit examples we show that without the geometric assumption on $Y$ such a bound can be arbitrarily far from holding.
\end{abstract}

\section{INTRODUCTION}

Let $Y$ be a closed, oriented 3-manifold. A contact structure on $Y$ is a 2 dimensional distribution of the form $\xi=\operatorname{ker} \theta \subset T Y$, where $\theta \in \Omega^{1}(Y)$ is a 1 -form on $Y$ such that $\theta \wedge d \theta>0$ at every point of $Y$. Note that, according to our definition, a contact structure $\xi=\operatorname{ker} \theta$ is equipped with a co-orientation given by $\theta$, an orientation given by the restriction of $d \theta$, and the two put together yield the given orientation on $Y$. Usually, such contact structures are called co-orientable and positive, but since we are not going to deal with their not co-orientable nor negative counterparts, the terminology will be abused throughout the paper. In any event, we refer the reader to [4] and 6] for a good introduction to contact structures.

By the work of Martinet and Lutz [13] every closed, oriented 3-manifold has a contact structure, but Eliashberg showed that certain contact structures which he called overtwisted are not so interesting. In fact, they are completely determined up to isotopy by the homotopy type of $Y$ [1]. On the other hand, not overtwisted or tight contact structures capture deeper information about $Y$ and are still quite mysterious.

A contact structure $\xi=\operatorname{ker} \theta$ on a closed oriented $3-$ manifold $Y$ is called symplectically fillable, or just fillable, if there exists a symplectic 4 -manifold $(X, \omega)$ such that $\partial X=Y$ as oriented manifolds while $\theta \wedge \omega>0$ at every point of $Y$. Fillable

Received by the editors November 29, 1999 and, in revised form, April 12, 2000.

2000 Mathematics Subject Classification. Primary 57M50, 57R57; Secondary 53C15, 57R15.

Key words and phrases. Contact structures, gauge theory, positive scalar curvature, symplectic fillings, Seiberg-Witten equations.

The author's research was partially supported by MURST. 
contact structures are tight [3, 11], while no example of a tight but not fillable contact structure is known at present.

The set $\mathcal{F}_{Y}$ of fillable contact structures considered up to homotopy (as cooriented 2-plane fields) on $Y$ is a subtle invariant of $Y$. By a theorem of Eliashberg and Thurston [5], the cardinality of $\mathcal{F}_{Y}$ is an upper bound for the number of homotopy classes of taut foliations on $Y$. Using a suitable version of the Seiberg-Witten equations, Kronheimer and Mrowka proved that $\mathcal{F}_{Y}$ is always a finite set [10]. This is the main general fact known about $\mathcal{F}_{Y}$. In this paper we build on the results of [10] and [12] to study the set $\mathcal{F}_{Y}$ under the assumption that $Y$ admits metrics with positive scalar curvature. The following is our main result.

Theorem 1.1. Let $Y$ be a closed oriented 3-manifold carrying metrics with positive scalar curvature. Then, two fillable contact structures on $Y$ are homotopic if and only if they are homotopic on the complement of a point.

By a result of Gompf ([8], Corollary 4.19), the conclusion of Theorem 1.1 holds for contact structures on spherical space forms with tight universal cover. On the other hand, as pointed out in [8], there exist many fillable contact structures on spherical space forms all of whose covers are overtwisted.

As we explain in section 2, the following is an immediate consequence of Theorem 1.1 .

Corollary 1.2. Let $Y$ be a closed oriented 3-manifold carrying metrics with positive scalar curvature. Then,

$$
\left|\mathcal{F}_{Y}\right| \leq \mid \text { Tor } H_{1}(Y ; \mathbb{Z}) \mid
$$

where Tor $H_{1}(Y ; \mathbb{Z})$ is the torsion subgroup of $H_{1}(Y ; \mathbb{Z})$ and || denotes cardinality.

Both Theorem 1.1 and Corollary 1.2 fail if one drops the geometric assumption on $Y$. In section 4 we show this by proving that if $Y_{n}, n \in \mathbb{N}$, is the integral homology 3 -sphere with the surgery presentation given in Figure 1 we have

$$
\left|\mathcal{F}_{Y_{n}}\right| \geq n .
$$

Therefore, the difference between $\left|\mathcal{F}_{Y}\right|$ and $\mid$ Tor $H_{1}(Y ; \mathbb{Z}) \mid$ can be arbitrarily large in general.

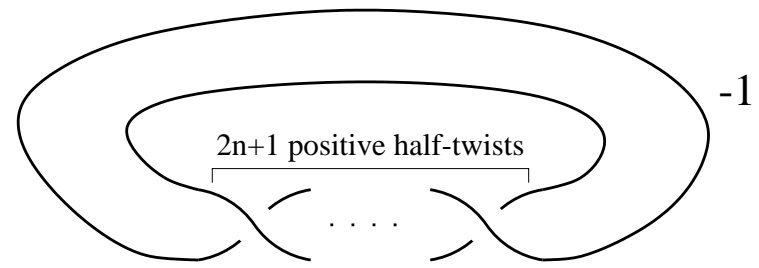

Figure 1. The homology 3-sphere $Y_{n}$

The paper is organized as follows. In section 2 we discuss the relationship between 2-plane fields and $\mathrm{Spin}^{c}$-structures, and we restate Theorem 1.1 in terms of the latter as Theorem [2.1] This is a natural intermediate step because Spin $^{c}$ structures are strictly related with the gauge theoretical techniques involved in the proof, given in section 3. In section 4 we quickly recall Eliashberg's Legendrian surgery construction and we apply it directly to prove (2). 
Recent work by Giroux [7] and by Honda [9] yields a classification of tight contact structures on lens spaces, showing in particular that all tight contact structures on lens spaces are fillable. The results of Giroux and Honda are consistent with Theorem 1.1, were obtained independently and do not rely on gauge theory.

\section{2-PLANE Fields AND $\operatorname{Spin}^{c}$ STRUCTURES}

In this section we recall the well-known relationship between oriented 2-plane fields and $\operatorname{Spin}^{c}$ structures. The discussion allows us to interpret the statement of Theorem 1.1 in terms of $\mathrm{Spin}^{c}$ structures, which are directly related to the SeibergWitten equations and the techniques of the next section. We restate Theorem 1.1 in this alternative language as Theorem [2.1, and we explain how to derive Corollary 1.2

Let $Y$ be a closed oriented 3-manifold. Due to the presence of the orientation on $Y$, a co-orientation of a 2-plane field $\xi$ on $Y$ is the same thing as an orientation on $\xi$. Hence, from now on we shall think of co-oriented 2-plane fields on $Y$ as oriented 2 -plane fields as well. Choose a Riemannian metric on $Y$ and let $\Xi$ denote the space of oriented 2-plane fields on $Y$. An oriented 2-plane field $\xi \in \Xi$ determines a lift of the structure group of the principal frame bundle $P_{S O(3)}$ of $Y$ from $S O(3)$ to $U(2)$, i.e. a $U(2)$-principal bundle $P_{U(2)}$ together with a principal bundle map $P_{U(2)} \rightarrow P_{S O(3)}=P_{U(2)} / U(1)$. Such a lift is called a Spinc structure on $Y$. The isomorphism class of the $\operatorname{Spin}^{c}$ structure determined by $\xi$ depends on $\xi$ only through its homotopy class $[\xi]$. Therefore, there is a natural map

$$
p: \pi_{0}(\Xi) \rightarrow \operatorname{Spin}^{c}(Y)
$$

and we set $\mathbf{t}_{\xi}=p([\xi])$.

Any trivialization of the frame bundle of $Y$ identifies $\pi_{0}(\Xi)$ with the set $\left[Y, S^{2}\right]$ of homotopy classes of maps from $Y$ to the 2 -sphere, and via the Pontryagin construction one can think of $\left[Y, S^{2}\right]$ as the set $\operatorname{Fr}_{Y}$ of framed 1-dimensional submanifolds of $Y$ up to framed cobordism. Moreover, a trivialization identifies $H^{2}\left(P_{S O(3)} ; \mathbb{Z}\right)$ with $H^{2}(Y ; \mathbb{Z}) \times \mathbb{Z} / 2 \mathbb{Z}$, and since $P_{U(2)} \rightarrow P_{S O(3)}$ restricts to the fibers of $P_{S O(3)} \rightarrow Y$ as the only non-trivial $U(1)$-bundle, the set $\operatorname{Spin}^{c}(Y)$ of $\operatorname{Spin}^{c}$ structures on $Y$ is identified with a copy of $H^{2}(Y ; \mathbb{Z})$ inside $H^{2}\left(P_{S O(3)} ; \mathbb{Z}\right)$. Therefore, via Poincaré duality a trivialization of the frame bundle of $Y$ identifies the set of $\operatorname{Spin}^{c}$ structures on $Y$ with $H_{1}(Y ; \mathbb{Z})$. Under the above identifications the map from $\operatorname{Fr}_{Y}$ to $H_{1}(Y ; \mathbb{Z})$ induced by $p$ sends a framed submanifold $\gamma_{[\xi]}$ - corresponding to the homotopy class $[\xi]$ - to the homology class $[\gamma] \in H_{1}(Y ; \mathbb{Z})$ supported by the underlying oriented submanifold, and $c_{1}(\xi)=2 \operatorname{PD}([\gamma]) \in H^{2}(Y ; \mathbb{Z})$. Moreover, it is not difficult to show that if $Y$ is connected, for every $\mathbf{t}_{\xi} \in \operatorname{Spin}^{c}(Y)$ the fiber $p^{-1}\left(\mathbf{t}_{\xi}\right)$ can be identified (in a non canonical way) with $\mathbb{Z} / d\left(\mathbf{t}_{\xi}\right) \mathbb{Z}$, where $d\left(\mathbf{t}_{\xi}\right) \in \mathbb{Z}$ is the divisibility of $c_{1}(\xi)$, defined to be zero if $c_{1}(\xi)$ is a torsion element (see e.g. [8], Proposition 4.1).

Let $\xi$ be a fillable contact structure on a closed oriented 3-manifold $Y$ carrying positive scalar curvature metrics. Then, $Y$ is connected and $c_{1}(\xi)$ is a torsion class [12]. In view of the previous discussion, the fiber $p^{-1}\left(\mathbf{t}_{\xi}\right)$ is a copy of $\mathbb{Z}$, so there exist infinitely many distinct homotopy classes of 2 -plane fields inducing the same $\operatorname{Spin}^{c}$ structure $\mathbf{t}_{\xi}$ on $Y$. In the next section we prove that the homotopy class of the fillable contact structure $\xi$ is the only homotopy class of oriented 2-plane fields, among the infinitely many inducing the same $\operatorname{Spin}^{c}$ structure, which can be realized by a fillable contact structure. This can be stated as follows. 
Theorem 2.1. Let $Y$ be a closed oriented 3-manifold carrying metrics with positive scalar curvature. Then, two fillable contact structures on $Y$ are homotopic if and only if they induce the same Spin ${ }^{c}$ structure on $Y$.

The statements of Theorems 1.1 and 2.1 are equivalent because, as explained e.g. in [15, the set $\operatorname{Spin}^{c}(Y)$ can be canonically identified with the set of nonvanishing vector fields on $Y$ up to homotopy on the complement of a ball. In view of the above discussion, Corollary 1.2 is an immediate consequence of Theorem 2.1

\section{The Seiberg-Witten equations}

In this section we prove Theorems 1.1 and 2.1 using techniques from [12]. We shall ultimately rely on results of Kronheimer and Mrowka; therefore we start by briefly recalling their set-up [10]. We refer the reader to the original paper for more details.

A Spin ${ }^{c}$ structure on a smooth oriented Riemannian $4-$ manifold $X$ is given by a triple $\left(W^{+}, W^{-}, \rho\right)$, where $W^{+}$and $W^{-}$are hermitian rank-2 bundles over $X$ called respectively the positive and negative spinor bundle, and $\rho: T^{*} X \rightarrow$ $\operatorname{Hom}\left(W^{+}, W^{-}\right)$is a map satisfying the Clifford relation: $\rho(\theta)^{*} \rho(\theta)=|\theta|^{2} \operatorname{Id}_{W^{+}}$ for every $\theta \in T^{*} X$. The map $\rho$ extends to a linear embedding $\rho: \Lambda^{*} T^{*} X \rightarrow$ $\operatorname{End}\left(W^{+} \oplus W^{-}\right)$. A Spin connection $A$ is a unitary connection on $W=W^{+} \oplus W^{-}$ such that the induced connection on $\operatorname{End}(W)$ agrees with the Levi-Civita connection on the image of $\rho$. To any Spin connection $A$ is associated, via $\rho$, a twisted Dirac operator $D_{A}^{+}: \Gamma\left(W^{+}\right) \rightarrow \Gamma\left(W^{-}\right)$.

A 4 -manifold with contact boundary is a pair $(X, \xi)$, where $X$ is a connected, oriented smooth 4-manifold with boundary and $\xi$ is a contact structure on $\partial X$ (endowed with the boundary orientation). Given a 4-manifold with contact boundary $(X, \xi)$, let $X^{+}$be the smooth manifold obtained from $X$ by attaching the open cylinder $[1,+\infty) \times \partial X$ along $\partial X$, and let $\theta \in \Omega^{1}(\partial X)$ with $\xi=\operatorname{ker} \theta$ and $\theta \wedge d \theta>0$ at every point of $\partial X$. On $X^{+}$there is a metric which restricts to $[1,+\infty) \times \partial X$ as a product metric of the form $d t^{2}+t^{2} g_{\partial X}$ ( $t$ being the coordinate function on the first factor), and such that the 2 -form $\omega_{0}=\frac{1}{2} d\left(t^{2} \theta\right)$ is self-dual of constant length $\sqrt{2}$. The choice of an $\omega_{0}$-compatible almost complex structure $J_{0}$ determines on $[1,+\infty) \times \partial X$ a $\operatorname{Spin}^{c}$ structure $\mathbf{s}_{0}$ and a unit section $\Phi_{0}$ of the corresponding positive spinor bundle. Moreover, there is a unique Spin connection $A_{0}$ such that $D_{A_{0}}^{+}\left(\Phi_{0}\right)=0$.

Let $\operatorname{Spin}^{c}(X, \xi)$ be the set of isomorphism classes of $\operatorname{Spin}^{c}$ structures on $X^{+}$ whose restriction to $[1,+\infty) \times \partial X$ is isomorphic to $\mathbf{s}_{0}$. Given $\mathbf{s} \in \operatorname{Spin}^{c}(X, \xi)$, use $\mathbf{s}$ to extend $\Phi_{0}$ and $A_{0}$ arbitrarily to all of $X^{+}$, and for every Spin connection $A$ for $\mathbf{s}$, let $\hat{A}$ be the induced $U(1)$ connection on the determinant of the positive spinor bundle. One can consider the set of pairs $(A, \Phi)$ satisfying the $\delta$-perturbed Seiberg-Witten equations

$$
\left\{\begin{array}{l}
\rho\left(F_{\hat{A}}^{+}\right)-\left\{\Phi \otimes \Phi^{*}\right\}=\rho\left(F_{\hat{A}_{0}}^{+}\right)-\left\{\Phi_{0} \otimes \Phi_{0}^{*}\right\}+\delta \\
D_{A}^{+}(\Phi)=0
\end{array}\right.
$$

where $A-A_{0}, \Phi-\Phi_{0}$ and $\delta$ belong to suitably defined Sobolev spaces, and where $\left\{\Phi \otimes \Phi^{*}\right\}$ denotes the traceless part of $\Phi \otimes \Phi^{*}$, thought of as a bundle endomorphism.

There is a gauge group acting on the solutions of (3) which is (a completion of) a space of maps from $X^{+}$to $S^{1}$ asymptotic to 1 as $t \rightarrow \infty$ on $[1,+\infty) \times \partial X$. The moduli space $M_{\delta}(\mathbf{s})$ is defined as the quotient of the set of solutions to (3) 
under the action of the gauge group. For $\delta$ in a Baire set of perturbing terms exponentially decaying along the end, $M_{\delta}(\mathbf{s})$ is a smooth, compact orientable manifold of dimension $d(\mathbf{s})=\left\langle e\left(W^{+}, \Phi_{0}\right),[X, \partial X]\right\rangle$, the obstruction to extending $\Phi_{0}$ as a non-vanishing section of $W^{+}$. Moreover, there is a map

$$
\mathrm{SW}_{(X, \xi)}: \operatorname{Spin}^{c}(X, \xi) \rightarrow \mathbb{Z}
$$

defined by setting $\mathrm{SW}_{(X, \xi)}(\mathbf{s})$ equal to the algebraic number of points of $M_{\delta}(\mathbf{s})$ when $d(\mathbf{s})=0$, and zero otherwise (strictly speaking, in order to specify the sign of the map $\mathrm{SW}_{(X, \xi)}$ one has to make a preliminary choice of an orientation on the moduli spaces). It turns out that the map $\mathrm{SW}_{(X, \xi)}$ does not depend on the choices involved, but only on the isomorphism class of the pair $(X, \xi)$. If $\omega$ is a compatible symplectic form on $(X, \xi)$, i.e. a symplectic form on $X$ such that $\left.\omega\right|_{\xi}>0$ at every point of $\partial X$, there is an induced $\operatorname{Spin}^{c}$ structure $\mathbf{s}_{\omega} \in \operatorname{Spin}^{c}(X, \xi)$ and

$$
\mathrm{SW}_{(X, \xi)}\left(\mathbf{s}_{\omega}\right)=1 \text {. }
$$

Proof of Theorems 1.1] and 2.1. By [12], if a closed 3-manifold carries positive scalar curvature metrics and fillable contact structures, then it cannot have more than one connected component. Therefore, it is not restrictive to assume that $Y$ is connected. At the end of section 2 we have observed that Theorems 1.1 and 2.1 are equivalent; hence we will prove only the statement of Theorem 2.1. One half of the statement is obvious because the discussion of section 2 immediately implies that two homotopic contact structures induce the same $\operatorname{Spin}^{c}$ structure. Therefore, we need to show that if $\xi$ is a fillable contact structure on $Y$, then its homotopy class is determined by $\mathbf{t}_{\xi} \in \operatorname{Spin}^{c}(Y)$.

Let $Y$ be the boundary of $X$, and let $g_{Y}$ be a positive scalar curvature metric on $Y$. Consider a Riemannian metric $g_{1}$ on $X^{+}$which coincides with $d t^{2}+t^{2} g_{Y}$ on $[1,+\infty) \times Y$ and such that $\left(X^{+}, g_{1}\right)$ contains an isometric copy of the cylinder $[-1,1] \times Y$ with the metric $d t^{2}+g_{Y}$. Denote by $X_{\text {in }}$ and $X_{\text {out }}$, respectively, the compact and non-compact component of the complement of the cylinder in $X^{+}$. Let $\widehat{X}_{\text {in }}$ and $\widehat{X}_{\text {out }}$ be, respectively, the Riemannian manifolds obtained by isometrically attaching cylinders $[-1,+\infty) \times Y$ and $(-\infty, 1] \times \bar{Y}$ with metric $d t^{2}+g_{Y}$ to $X_{\text {in }}$ and $X_{\text {out }}$ respectively, where $\bar{Y}$ denotes $Y$ with the opposite orientation. Let $\delta_{\text {in }}$ and $\delta_{\text {out }}$ be compactly supported perturbing terms on $\widehat{X}_{\text {in }}$ and $\widehat{X}_{\text {out }}$ respectively. Denote the corresponding moduli spaces of solutions with bounded variation of the CSD functional along the end by $M_{\delta_{\text {in }}}\left(\widehat{X}_{\text {in }}\right)$ and $M_{\delta_{\text {out }}}\left(\widehat{X}_{\text {out }}, \xi\right)$.

The results of [14] imply that $\left(A_{\text {in }}, \phi_{\text {in }}\right)$, restricted to the slices $\{t\} \times Y$, converges, as $t \rightarrow+\infty$, towards an element of the moduli space $N_{X}\left(Y, \mathbf{t}_{\xi}\right)$ of solutions of the unperturbed 3-dimensional monopole equations on $Y$ (corresponding to the $\operatorname{Spin}^{c}$ structure $\mathbf{t}_{\xi}$ ) modulo gauge transformations which extend over $X$. This implies that there is a map $\partial_{X_{\text {in }}}: M_{\delta_{\text {in }}}\left(\widehat{X}_{\text {in }}\right) \rightarrow N_{X_{\text {in }}}\left(Y, \mathbf{t}_{\xi}\right)$, and for every $\theta \in N_{X_{\text {in }}}\left(Y, \mathbf{t}_{\xi}\right)$, we denote $\partial_{X_{\text {in }}}^{-1}(\theta)$ by $M_{\delta_{\text {in }}}\left(\widehat{X}_{\text {in }}, \theta\right)$. Similarly, there is a map $\partial_{X_{\text {out }}}: M_{\delta_{\text {out }}}\left(\widehat{X}_{\text {out }}\right) \rightarrow N_{X_{\text {out }}}\left(\bar{Y}, \mathbf{t}_{\xi}\right)$, and for $\theta^{\prime} \in N_{X_{\text {out }}}\left(\bar{Y}, \mathbf{t}_{\xi}\right)$ we denote $\partial_{X_{\text {out }}}^{-1}\left(\theta^{\prime}\right)$ by $M_{\delta_{\text {out }}}\left(\widehat{X}_{\text {out }}, \xi, \theta^{\prime}\right)$.

If $(X, \omega)$ is a symplectic filling of $(Y, \xi)$, then by (4) we have $\mathrm{SW}_{(X, \xi)}\left(\mathbf{s}_{\omega}\right) \neq 0$. By [12], this implies that for any $\theta_{1} \in N_{X_{\text {in }}}\left(Y, \mathbf{t}_{\xi}\right), \theta_{2} \in N_{X_{\text {out }}}\left(\bar{Y}, \mathbf{t}_{\xi}\right)$, if $d_{1}$ and $d_{2}$ denote, respectively, the expected dimensions of $M_{\delta_{\text {in }}}\left(\widehat{X}_{\text {in }}, \theta_{1}\right)$ and $M_{\delta_{\text {out }}}\left(\widehat{X}_{\text {out }}, \xi, \theta_{2}\right)$, we have $d_{1}+d_{2}=-1-b_{1}(Y)$ while $d_{2} \geq 0$. Moreover, it follows from the AtiyahPatodi-Singer index theorem that the difference $d_{1}-\frac{1}{4}\left(c_{1}\left(W_{X}^{+}\right)^{2}-2 \chi(X)-3 \sigma(X)\right)$ 
only depends on the Riemannian manifold $Y$ and the $\operatorname{Spin}^{c}$ structure $\mathbf{t}_{\xi}$ (see [12] for a formula involving an eta invariant). Note that the rational number $c_{1}\left(W_{X}^{+}\right)^{2}$ is well defined because, by Proposition 2.1 of [12], $\left.c_{1}\left(W_{X}^{+}\right)\right|_{Y}$ is a torsion class.

We claim that $d_{2}$ cannot be greater than zero. Assuming this fact for the moment, let us see why it implies the statement. In view of the results mentioned in the previous paragraph, the claim clearly implies that $d_{2}=0$ and $d_{1}=-1-b_{1}(Y)$. Therefore, the number $c_{1}\left(W_{X}^{+}\right)^{2}-2 \chi(X)-3 \sigma(X)$ only depends on the pair $\left(Y, \mathbf{t}_{\xi}\right)$, and since the $\operatorname{Spin}^{c}$ structure on $X$ is induced by an almost complex structure $J_{X}$, we have $c_{1}\left(W_{X}^{+}\right)=c_{1}\left(J_{X}\right)$. On the other hand, by e.g. [8], the number $c_{1}\left(J_{X}\right)^{2}-2 \chi(X)-3 \sigma(X)$ determines the homotopy class $[\xi] \in p^{-1}\left(\mathbf{t}_{\xi}\right)$. This proves the statement assuming the claim.

Let us turn to the proof of the claim. The moduli space $M_{\delta_{\text {out }}}\left(\widehat{X}_{\text {out }}, \xi, \theta_{2}\right)$ sits inside the quotient $\mathcal{B}=\mathcal{C} / \mathcal{G}$, where $\mathcal{C}$ is a configuration space of pairs $(A, \Phi)$ and $\mathcal{G}$ is the gauge group. Observe that $\mathcal{C}$ is contractible being the cartesian product of an affine space by a vector space. The gauge group $\mathcal{G}$ is contractible as well, because by definition its elements are maps from $X^{+}$to the circle which are asymptotic to 1 towards $+\infty$. Moreover, $\mathcal{G}$ acts freely on $\mathcal{C}$ because, for every $(A, \Phi) \in \mathcal{C}, \Phi$ is asymptotic to $\Phi_{0}$ towards $+\infty$. Therefore, since $\mathcal{B}$ is the base of a fibration whose fiber and total space are both contractible, $\mathcal{B}$ itself is contractible.

Arguing by contradiction, let us assume that $d_{2}>0$. Since the moduli space $M_{\delta}\left(\mathbf{s}_{\omega}\right)$ is smooth and 0-dimensional, this would imply by standard gluing theory that there is an obstruction to extending solutions of the Seiberg-Witten equations from $X_{\text {out }}$ to $X^{+}$. Since the character variety of $Y$ is smooth, this means that $M_{\delta}\left(\mathbf{s}_{\omega}\right)$ can be identified with the zero set of a generic section of an obstruction bundle $\mathcal{O} \rightarrow M_{\delta_{\text {out }}}\left(\widehat{X}_{\text {out }}, \xi, \theta_{2}\right)$, where $\mathcal{O}$ is the restriction of an oriented bundle over $\mathcal{B}$. Therefore $\mathrm{SW}_{(X, \xi)}\left(\mathbf{s}_{\omega}\right)$, which is the algebraic number of points in $M_{\delta}\left(\mathbf{s}_{\omega}\right)$, equals

$$
\left\langle e(\mathcal{O}),\left[M_{\delta}\left(\widehat{X}_{\text {out }}, \xi, \theta_{2}\right)\right]\right\rangle
$$

where $e(\mathcal{O})$ denotes the Euler class of $\mathcal{O}$. This number is zero, because $\mathcal{O}$ is the restriction of a bundle over the contractible space $\mathcal{B}$. We have concluded that $d_{2}>0$ implies $\mathrm{SW}_{(X, \xi)}\left(\mathbf{s}_{\omega}\right)=0$, in contradiction with (4). This establishes the claim and concludes the proof of Theorems 1.1 and 2.1.

\section{LEGENDRIAN SURGERIES}

In this section we prove the inequality (21). We start by briefly recalling a particular case of Eliashberg's Legendrian surgery construction. See [2] or [8] for generalizations and details.

A Legendrian knot $K$ in a contact manifold $(Y, \xi)$ is a smooth knot in $Y$ which is everywhere tangent to $\xi$. The distribution $\xi$ determines, in an obvious way, a framing of $K$ which we shall call the contact framing.

The standard contact structure $\xi_{0}$ on the 3 -sphere $S^{3} \subset \mathbb{C}^{2}$ is the oriented distribution given by the tangent complex lines. Given a Legendrian knot $K$ inside $\left(S^{3}, \xi_{0}\right)$, when the contact framing of $K$ is identified in the usual way with an integer, it coincides with an invariant of the Legendrian isotopy class of $K$, the Thurston-Bennequin number $\operatorname{tb}(K) \in \mathbb{Z}$. Eliashberg's Legendrian surgery says, in particular, that by attaching a 2 -handle to $K$ with framing $\operatorname{tb}(K)-1$ one obtains a Stein 4-manifold with boundary $X$, which is a special kind of symplectic filling 
with exact symplectic form. What is interesting for us is the fact that there are a contact structure $\zeta$ on $\partial X$ and a symplectic form on $X$ which is positive on $\zeta$. The first Chern class $c_{1}(X) \in H^{2}(X ; \mathbb{Z})$ can be easily computed from $K$ : the value of the pairing of $c_{1}(X)$ with the 2-homology class of $X$ determined by the oriented knot $\vec{K}$ is equal to the value of another well-known invariant of the Legendrian isotopy class of $K$, the rotation number $\operatorname{rot}(\vec{K}) \in \mathbb{Z}$ (see below for a definition).

Now we need to recall how to represent a Legendrian knot $K$ in $\left(S^{3}, \xi_{0}\right)$ and how to compute $\operatorname{tb}(K)$ and $\operatorname{rot}(\vec{K})$. When restricted to the complement of a point, $\xi_{0}$ is isomorphic to the contact structure on $\mathbb{R}^{3}$ defined by the 1 -form $\theta=d z+x d y$. Therefore, without loss of generality we can assume that $K$ sits inside $\mathbb{R}^{3}$ with the contact structure given by the kernel of $\theta$. Up to a small Legendrian perturbation, the projection of $K$ to the $y z$-plane is a front, that is, a piecewise-smooth immersed curve with finitely many singularities which are either transversal double points or horizontal cusps. Moreover, the cusps are exactly the local extrema of the restriction of the coordinate function $y$. Using the differential equation $d z / d y=-x, K$ can be reconstructed from its front projection, and a knot diagram for $K$ is easily obtained by letting the over-arc at any double point be the one with the most negative slope. Let $w$ denote the writhe of the diagram, i.e. the algebraic number of self-intersections, and $c$ the number of cusps. Then, $\operatorname{tb}(K)=w-\frac{1}{2} c$. If $\vec{K}$ denotes an oriented copy of $K$, let $a$ be the number of ascending cusps of its front and $d$ the number of descending cusps. Then, $\operatorname{rot}(\vec{K})=\frac{1}{2}(d-a)$.

Proof of inequality (2n). Figure2 2 represents the oriented fronts $F_{n, h}$ which will yield our examples via Legendrian surgery. Let $\vec{K}_{n, h}$ be the oriented Legendrian knot corresponding to the oriented front $F_{n, h}$ of Figure 2, $1 \leq h \leq 2 n$. From the front one computes $\operatorname{tb}\left(K_{n, h}\right)=0$ and $\operatorname{rot}\left(\vec{K}_{n, h}\right)=1+2(n-h)$. Let $X_{n, h}$ be the Stein manifold with boundary constructed by attaching a 2-handle along $K_{n, h}$. We have $c_{1}\left(X_{n, h}\right)=-(1+2(n-h)) \operatorname{PD}(g)$, where $g \in H_{2}\left(X_{n, h} ; \mathbb{Z}\right) \cong \mathbb{Z}$ is the generator corresponding to the oriented knot $\vec{K}_{n, h}$.

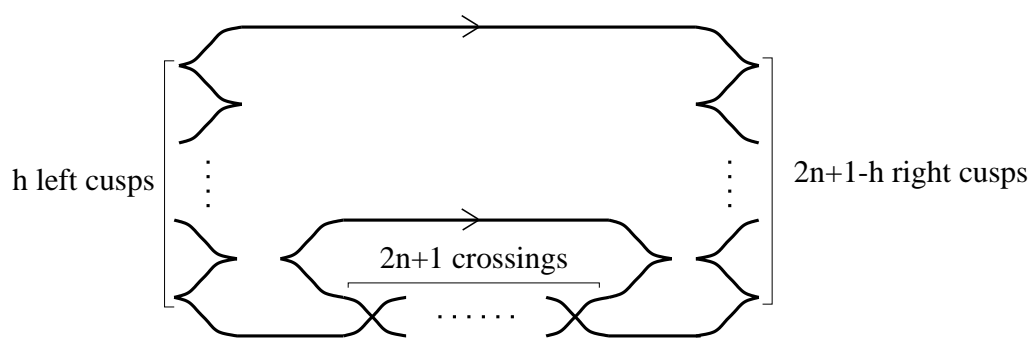

Figure 2. The oriented front $F_{n, h}$

Denote by $X_{n}$ the smooth 4-manifold underlying $X_{n, h}$, and let $Y_{n}=\partial X_{n}$. Let $\xi_{n, h}$ be the fillable contact structure induced on $Y_{n}$ by $X_{n, h}$. By [8], the homotopy class of $\xi_{n, h}$ can be identified with the integer $c_{1}\left(X_{n, h}\right)^{2}-2 \chi\left(X_{n}\right)-3 \sigma\left(X_{n}\right)=$ $(1+2(n-h))^{2}-1$. It follows that the contact structures $\xi_{n, 1}, \xi_{n, 2}, \ldots, \xi_{n, n}$ represent $n$ different homotopy classes on $Y_{n}$. 


\section{REFERENCES}

[1] Y. Eliashberg, Classification of overtwisted contact structures on 3-manifolds, Invent. Math. 98 (1989), 623-637. MR 90k:53064

[2] _ Topological characterization of Stein manifolds of dimension $>2$, Intern. J. of Math. 1, No. 1 (1990), 29-46. MR 91k:32012

[3] — Filling by holomorphic discs and its applications, in "Geometry of lowdimensional manifolds", 2 (Durham, 1989), London Math. Soc. Lecture Notes Series 151 (1991), 45-67. MR 93g:53060

[4] - Contact 3-manifolds twenty years since J. Martinet's work, Ann. Inst. Fourier 42 (1992), 165-192. MR 93k:57029

[5] Y. Eliashberg, W. Thurston, Confoliations, AMS University Lecture Series 13, Providence, RI, 1998. MR 98m:53042

[6] E. Giroux, Topologie de contact en dimension 3 (autour des travaux de Yakov Eliashberg), Séminaire Bourbaki, Vol. 1992/93. Astérisque No. 216, (1993), Exp. No. 760, 3, 7-33. MR 94k:57040

[7] _ Structures de contact en dimension trous et bifurcations des feuilletages de surfaces, preprint, 1999.

[8] R.E. Gompf, Handlebody construction of Stein surfaces, Ann. of Math. (2) 148 (1998), no. 2, 619-693. MR 2000a:57070

[9] K. Honda, On the Classification of Tight Contact Structures I: Lens Spaces, solid Tori, and $T^{2} \times I$, preprint, 1999 .

[10] P.B. Kronheimer, T.S. Mrowka, Monopoles and contact structures, Invent. Math. 130 (1997), 209-256. MR 98h:57058

[11] F. Laudenbach, Orbites périodiques et courbes pseudo-holomorphes, application à la conjecture de Weinstein en dimension 3 [d'après H. Hofer et al.], Astérisque 227 (1995), 309-333. MR 96c:58065

[12] P. Lisca, Symplectic fillings and positive scalar curvature, Geometry and Topology 2 (1998), 103-116. MR 99f:57038

[13] J. Martinet, Formes de contact sur les variètès de dimension 3, Lecture Notes in Math. 209, Springer-Verlag (1971), 142-163. MR 50:3263

[14] J.W. Morgan, T.S. Mrowka and D. Ruberman, The $L^{2}-$ moduli space and a vanishing theorem for Donaldson polynomial invariants, Monographs in Geometry and Topology, no. II, International Press, Cambridge, MA, 1994. MR 95h:57039

[15] V. Turaev, Torsion invariants of Spin ${ }^{c}$ structures on 3-manifolds, Math. Res. Lett. 4 (1997), no. 5, 679-695. MR 98k:57038

Dipartimento di Matematica, Università di Pisa I-56127 Pisa, Italy

E-mail address: lisca@dm.unipi.it

URL: http://www.dm.unipi.it/pages/lisca/public_html 